\title{
A Comparative Study of Damage Performance of the Kill Element from Different Materials
}

\author{
J. P. Yin, ${ }^{1}$ B. B. Gao, Z. J. Wang, and C. L. Zhao \\ School of Mechatronic Engineering, North University of China, Taiyuan, China \\ 1yjp123@nuc.edu.cn
}

For the design of the novel anti-explosive reactive armor tandem warhead, a prerequisite is to improve the reaming capacity and the damage performance of the preceding kill element so that the channel is ready for the subsequent penetration by the kill element. Meanwhile, the selection of appropriate shaped charge liner material in the warhead could help enhance the integrated penetration performance of the kill element. Traditional shaped-charge liners made of metals or metal alloys with high density, high sound speed and good ductility are capable of forming a good-shape and stable jet kill element, which also demonstrate the advantages of large impact and high-performance penetration depth against the target. When the traditional liners are used to impact reactive armor, however, the weak reaming capacity and easily-induced charge explosions prevent the subsequent penetration of kill element into the main armor. In addition, the jet kill element formed by shaped-charge liners with low-density materials generally displays a low penetration depth against the reactive armor. In the present study, filled modified polytetrafluoroethylene (PTFE) was selected as the material of the shaped charge liner. The damage performances on the armor from the kill elements formed with metallic or nonmetallic liners were evaluated and compared based on the numerical simulations and experimental studies. The results showed that the head diameter of the PTFE-Cu jet kill element was increased by $11.1 \%$ as compared to the PTFE jet kill element, and the former was twice as large as that of the copper jet kill element. The stronger reaming capacity against the target was essential for the opening of a channel for the tandem warhead's subsequent element. In addition, when compared to the PTFE jet kill element, the penetration depth and the jet hole diameter of the PTFE-Cu one were increased by 45.8 and $12.6 \%$, respectively, demonstrating the high damage potential of the PTFE-Cu jet kill element. Therefore, the present comparative analysis of the kill element damage performance with different materials under high-speed impact loading has provided a reference for the research and the design of the anti-armor tandem warhead with large penetration apertures and high damage performance.

Keywords: explosion mechanics, penetrator, X-ray test, numerical simulation, damage efficiency.

Introduction. In the modern anti-armor warfare, the issues of overcoming the armored targets with wrap-type explosive reactive armor and improving the overall penetration performance of the shaped charge jet kill element remain topical [1]. Previous studies $[2,3]$ suggest that the key to solving such problem is to control the length, shape, mass, energy and several other parameters of the kill element, among which the selection of the right liner materials is essential, as suitable line materials can improve the integrated penetration performance of the kill element [4]. Striving to find the appropriate shaped charge liner materials, researchers pursue advanced materials with high density, subsonic and sonic speeds, dynamic elongation rate and non-vaporization properties. Several metals, such as copper, tungsten, molybdenum, and nickel, have been widely used as shaped charge liner materials. Hu et al. [5] studied the shaped charge material mechanical properties of $\mathrm{Mo}, \mathrm{Ta}$, and $\mathrm{W}$, and found that these metals and their alloys generally had high densities, high or moderate speeds of sound, good dynamic elongation rate, and could form ductile kill elements under explosive loading. Furthermore, the penetration performances of the kill element with Mo, Ta, and $\mathrm{W}$ addition were significantly improved as compared to the kill element with conventional copper liner material. Guo et al. [6] studied the development 
status of the shaped charge liner materials, and discovered that the liner material plasticity, density and sound speed were directly related to its penetration performance. Wu et al. [7] studied the shaped charge with the liner material of a powder-type tungsten alloy, and found that the penetration aperture could be greatly enhanced. Obviously, the traditional shaped charge that adopts metals, metal alloys and high-density materials could form continuous and stable kill elements with a certain length. Although it shows the advantage of large impact and great penetration depth against the target, the shortcomings include weak reaming capacity, small penetration aperture and limited lethality. At present, the design requirement for the new generation anti-explosive reactive armor's tandem warhead is to improve the reaming capacity of the kill element with a certain penetration depth as a prerequisite, and to open channels for the kill element subsequent target penetration. Therefore, studies $[8,9]$ on the use of low-density material as shaped charge liner material to achieve the goal of large perforations against the target have become a major research direction of anti-armor munitions. Ma et al. [10] adopted non-metallic graphite, ceramics and plexiglas as liner materials, and found that these materials formed jet kill elements with limited penetration capability, and often failed to achieve the maximum penetration. Dong et al. [11] adopted low-density materials (e.g., nylon, resin glass and titanium) to form shaped-charge jet. The results showed that the shaped charge kill element using these materials generally displayed a certain degree of penetration ability, but the overall penetration and reaming capability was not ideal due to very low density of the kill element $[12,13]$. To solve these problems, the present study proposes to use filled modified PTFE [14] (PTFE-Cu) as the shaped charge liner material, and analyzes the formation and damage performance of corresponding kill element. In addition, armor target penetration performance using the kill elements with the proposed liner or other materials is evaluated and compared based on numerical simulations and experimental studies. In other words, the damage performances of the kill element against the armor with different materials under high-speed impact loading are studied. Therefore, the present study provides a necessary reference to the research and the design of the anti-armor tandem warhead with large penetration apertures and high damage performance.

\section{Materials}

1. Finite Element Modeling of the Kill Element Formation for Different

1.1. Geometrical Model. In this study, the penetration level into steel 45 targets using the kill element formed by $40 \mathrm{~mm}$ caliber warhead was studied. The structure of the shaped charge was taper liner with uniform wall thickness, and the taper top was fillet processed. The initial parameters were: the cone angle of the liner was $55^{\circ}$, the charge height was $40 \mathrm{~mm}$, the charge mass was $60 \mathrm{~g}$, and the explosion height was $130 \mathrm{~mm}$. In order to test the damage performance of the PTFE- $\mathrm{Cu}$ jet kill element, liner warheads combined with copper, PTFE and PTFE-Cu material, respectively, were adopted to form jet kill element with the same shaped charge structure, and their penetration against steel 45 targets were studied accordingly. The wall thickness of the copper liner was $1.1 \mathrm{~mm}$, and that of PTFE and PTFE-Cu liners was $3.5 \mathrm{~mm}$.

1.2. Constitutive Relations and Parameters of the Main Materials. In numerical simulation, the used liner materials included copper, PTFE and PTFE-Cu, and the target plate material was steel 45 . Charge " $\mathrm{B}$ " was adopted. The constitutive relations and the parameters [15] of the main materials are shown in Tables 1 and 2. In Table 2, $\rho_{0}$ is density, $D$ is the detonation velocity of charge $\mathrm{B}$, while $A, B, R_{1}, R_{2}$, and $\omega$ are the input parameters under the dynamic test conditions, $E_{0}$ is the initial ratio of the internal energy, and $P_{C-J}$ is $\mathrm{C}-\mathrm{J}$ pressure.

1.3. Finite Element Model. Since large-scale deformation of materials occurrs when warhead explosion forces the liner to form a kill element, the Euler algorithm is applied to handle the large-scale deformation problem in the warhead region. On the other hand, the Lagrangian algorithm is applied to the target region. These two algorithms are also 
$\mathrm{T}$ a b 1 e 1

Constitutive Relations and Basic Material Parameters

\begin{tabular}{|c|c|c|c|c|}
\hline \multirow[t]{2}{*}{ Material } & \multirow{2}{*}{$\begin{array}{l}\text { Density } \\
\left(\mathrm{g} / \mathrm{cm}^{3}\right)\end{array}$} & \multirow[t]{2}{*}{ State equation } & \multicolumn{2}{|c|}{ Strength model } \\
\hline & & & $G, \mathrm{GPa}$ & $\sigma_{Y}, \mathrm{MPa}$ \\
\hline $\mathrm{Cu}$ & 8.93 & Shock & 46.00 & 90 \\
\hline PTFE & 2.16 & Shock & 2.33 & 50 \\
\hline PTFE-Cu & 3.05 & Shock & 1.37 & 46 \\
\hline 45 steel & 7.85 & Linear & 81.80 & 352 \\
\hline
\end{tabular}

T a b 1 e 2

Basic Parameters of Warhead Charge

\begin{tabular}{|c|c|c|c|c|c|c|c|c|}
\hline $\begin{array}{c}\rho_{0}, \\
\mathrm{~g} / \mathrm{cm}^{3}\end{array}$ & $\begin{array}{c}D, \\
\mathrm{~km} / \mathrm{s}\end{array}$ & $\begin{array}{c}A, \\
\mathrm{MPa}\end{array}$ & $\begin{array}{c}B, \\
\mathrm{MPa}\end{array}$ & $R_{1}$ & $R_{2}$ & $\omega$ & $\begin{array}{c}E_{0}, \\
\mathrm{~kJ} / \mathrm{m}^{3}\end{array}$ & $\begin{array}{c}P_{C-J}, \\
\mathrm{GPa}\end{array}$ \\
\hline 1.717 & 7.98 & 524230 & 7678 & 44.2 & 11.1 & 0.34 & $8.5 \cdot 10^{6}$ & 29.5 \\
\hline
\end{tabular}

integrated using the fluid-solid coupling method. In order to improve the calculation accuracy, structured grids were adopted and FLOUOUT was used as the boundary condition. In order to improve computational efficiency and to save shaped charge resources, gradient meshes with intermediate regions encrypted were adopted in the Euler domain. The target was modelled using uniform grid with two grids per mm. When copper was used as the liner material to form kill element, the penetration depth was significantly larger than the scenario when non-metallic kill elements were used due to high density of kill element in the former case. Therefore, a thicker target plate was established for copper warhead in finite element modeling, and the target plate was split into two sections. The length of the target plate penetrated by the copper kill element was 100 and $50 \mathrm{~mm}$ from the top and bottom, respectively. On the other hand, the length of the target plate penetrated by the PTFE kill element and the PTFE-Cu kill element was 50 and $50 \mathrm{~mm}$ from the top and bottom, respectively. The finite element model of the target penetration of the kill element formed by the warhead was constructed.

\section{Numerical Simulation of Kill Element Formation with Different Materials.}

2.1. The Formation and Velocity Cloud Images of Kill Elements with Different

Materials. Finite element models were established according to above-mentioned parameters, and the numerical simulation results showed that the kill element formed using the liners of three different types of materials generally displayed similar patterns as is shown in Fig. 1. The explosive detonation wave first reached the top of the liner while initiating, and then began to act on the liner after $3 \mu \mathrm{s}$. Due to the effect of detonation wave, the liner moved closely to the central axis after $5 \mu \mathrm{s}$. After the liner axis collision, a jet head started to form, and the basic form of the jet would form after $20 \mu \mathrm{s}$. Since the jet head speed was significantly higher than the tail one, the jet was gradually stretched and attenuated with time. Afterwards, slight differences among the jet kill elements with three different kinds of materials would appear. Necking phenomenon in the jet firstly appeared in the copper jet, then in the PTFE one, and finally in the PTFE-Cu one. In spite of the necking phenomenon, the jet was not pulled off yet. Subsequently, the copper jet was firstly pulled off until $45 \mu \mathrm{s}$, and the PTFE jet was then pulled off at $50 \mu \mathrm{s}$. However, the PTFE-Cu jet was not pulled off even at $60 \mu$ s. Therefore, the superior ductility of the PTFE-Cu jet was sufficiently demonstrated, which yielded valuable information for the improvement of its penetration performance. The velocity cloud images of the PTFE- $\mathrm{Cu}$ and PTFE jet kill element showed that there was a uniform velocity gradient in the kill element, which was 
uniformly stretched as time went by. The PTFE-Cu liner and the PTFE liner were capable of forming well-shaped jet kill element. Furthermore, they displayed certain extension characteristics and, thus, could not be easily pulled off. As a result, they are quite promising liner materials. As can be seen from the jet formation process at different phases, after the same duration, the PTFE-Cu jet kill element has a small slug, a good-shape head and good consistency in the jet tensile process, mainly due to higher density and lower shear modulus of PTFE-Cu as compared to the PTFE.

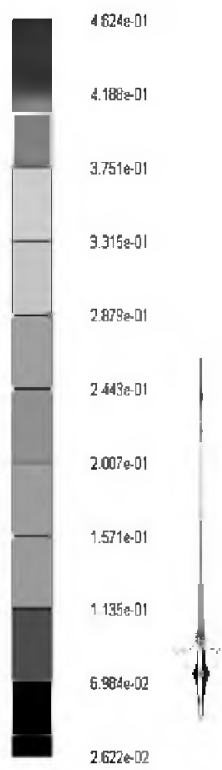

a

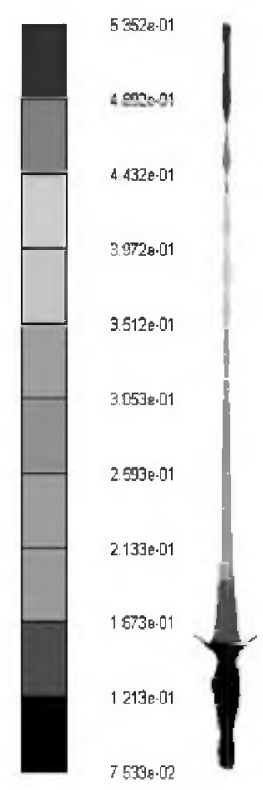

b

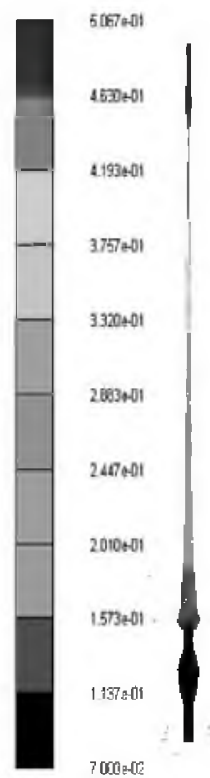

c

Fig. 1. The formation and velocity cloud images of kill elements with different materials: $\mathrm{Cu}$ (a). PTFE (b), and PTFE-Cu (c) jets.

\subsection{Numerical Simulation Results and Analysis of the Kill Element Formation.}

Numerical simulation of the jet formation process comprises three processes, including the shaped charge detonation, liner crush, and jet formation and stretching. These three processes are all axisymmetric. In the following analysis, the length, head diameter, and head speed of the jet kill element were used as the indices, and the kill element performances when the liners were formed with these three different materials were comparatively analyzed. Since the densities of the materials are significantly different (PTFE $<$ PTFE- $\mathrm{Cu}<\mathrm{Cu}$ ), with the same shaped charge structure and duration under the impact of detonation, the PTFE material formed the longest jet flow, whereas the copper material formed the shortest jet flow, with the PTFE-Cu material in between. This indicated that the plastic deformation of PTFE was larger than that of PTFE- $\mathrm{Cu}$, and the material utilization of the copper liner was the most inadequate. Since the shear stress generated from the interlayer velocity difference in the copper linear was less than the shear strength of the material, most liners converged into a symmetrical bullet shape. As can be seen from Table 1, since the compression strength of the PTFE-Cu was greater than that of PTFE, the PTFE material could form longer kill element at a faster pace under detonation pressure. The stretching rate of the PTFE-Cu kill element was smaller than that of PTFE. Before the kill element was pulled off ( $50 \mu \mathrm{s})$, the kill element lengths of PTFE-Cu and PTFE were 226 and $238 \mathrm{~mm}$, respectively. The PTFE kill element was 5.31\% longer than the PTFE-Cu one. 
As can be seen from the stretch length history diagram of the kill element (Fig. 2a), the kill element formed by PTFE was slightly longer than the PTFE-Cu one when the shaped charge structure and duration remained the same under the impact of detonation. With the passage of time, the length difference first increased and then decreased. Finally, there was almost no detectable difference in length at the time of $60 \mu \mathrm{s}$. From Fig. 2b, it can be seen that the head diameter of the copper jet kill element was the smallest after the jet kill element became stable when the shaped charge structure and duration remained the same under the impact of detonation. The kill element head diameter formed by PTFE was smaller than the PTFE-Cu kill element. The kill element head diameter no longer changed with time: after $20 \mu \mathrm{s}$, it remained in the steady state. The kill element head diameters of the PTFE-Cu and the PTFE were 3 and $2.7 \mathrm{~mm}$, respectively. The PTFE-Cu kill element head diameter was larger by $11.1 \%$ than that of PTFE one, and was twice that of the copper one. Since the diameter of the PTFE-Cu head was the largest, it should have the higher reaming capability against the target, or for the opening of the channel for the tandem warhead subsequent penetration.

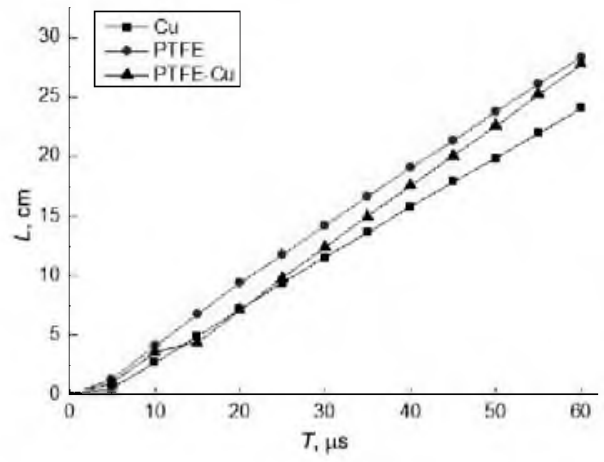

a

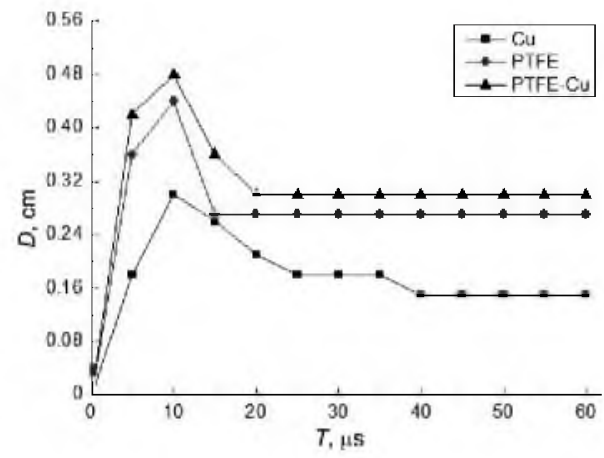

b

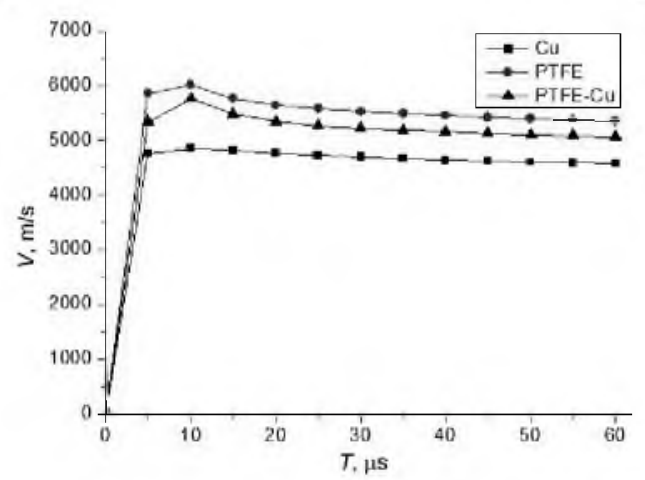

c

Fig. 2. Evolution of the kill element parameters for different materials: (a) length $L$; (b) head diameter $D$; (c) head speed $V$.

From Fig. 2c, it can be seen that the act of the detonation pressure on the shaped charge liner resulted in the crush of the liner and rapid formation of the kill element head. At about $10 \mu \mathrm{s}$, the kill element maximum head speed reached 6021,5766 , and $4860 \mathrm{~m} / \mathrm{s}$ for PTFE, PTFE-Cu, and $\mathrm{Cu}$, respectively. Subsequently, during the gradual stretching process of the kill element, a velocity gradient was formed between the kill element head and the slug. However, there was still a relatively high penetration speed of the reactive armor, and the penetration could be effectively conducted. At $40 \mu \mathrm{s}$, the kill element head 
speed was 5455,5158 , and $4642 \mathrm{~m} / \mathrm{s}$ for PTFE, PTFE $-\mathrm{Cu}$, and $\mathrm{Cu}$ jets, respectively. The PTFE - Cu kill element head speed was higher by $11.1 \%$ than that of the $\mathrm{Cu}$ one and, thus, was significantly improved. On the other hand, the PTFE-Cu kill element head speed was by $3.5 \%$ lower than that of PTFE one. Because the amplitude of the reduction was relatively small, it was helpful for the improvement of deep penetration capability.

\section{Numerical Simulation of Target Penetration by Kill Element from Different Materials.}

3.1. Jet Kill Element Penetration into Different Targets. To further study the penetration capability of the kill elements when combined with three different materials, the finite element model was established to calculate the penetration capability against the steel 45 armor. Starting with the warhead detonated, the copper jet kill element head started to penetrate the steel 45 target plate with a speed of $4743 \mathrm{~m} / \mathrm{s}$ at the time of $T=31 \mu \mathrm{s}$. The PTFE jet kill element started to penetrate the target plate with a speed of $5575 \mathrm{~m} / \mathrm{s}$ at the time of $T=25 \mu \mathrm{s}$, which was $6 \mu$ s earlier than the copper jet kill element. The PTFE $\mathrm{Cu}$ jet kill element penetration into the target plate with a speed of $5263 \mathrm{~m} / \mathrm{s}$ started at the time of $T=27 \mu \mathrm{s}$, which was by $2 \mu$ s later than that of PTFE one. The penetration process is shown in Fig. 3.

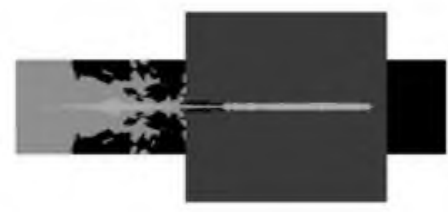

a

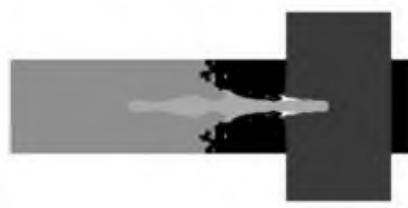

b

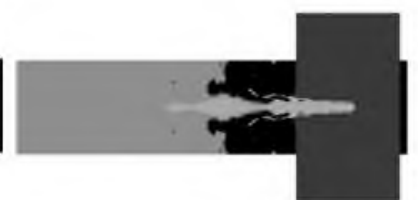

$\mathrm{C}$

Fig. 3. The process target penetration by the kill element jet from different materials: Cu (a), PTFE (b), and PTFE Cu (c).

3.2. Comparative Analysis of Jet Penetration Results for Different Materials. The numerical simulations of jet formation and penetration against the steel 45 by shaped charge liner with the three materials demonstrated that the copper jet had the lowest head speed but the highest penetration capability, with a penetration depth of $98.4 \mathrm{~mm}$. The PTFE jet head speed reached $5575 \mathrm{~m} / \mathrm{s}$, with the penetration depth as $22.1 \mathrm{~mm}$. The PTFE $-\mathrm{Cu}$ jet head speed reached $5263 \mathrm{~m} / \mathrm{s}$, and the penetration depth was $30.3 \mathrm{~mm}$. Overall, due to the material modification of PTFE, the PTFE- $\mathrm{Cu}$ jet head speed was reduced by $5.6 \%$ as compared to the PTFE one. The head diameter of PTFE-Cu jet was $3.2 \mathrm{~mm}$, while the diameter of PTFE jet was $2.7 \mathrm{~mm}$. The head diameter of PTFE-Cu jet was increased by $18.5 \%$ compared to the PTFE jet. The PTFE $\mathrm{Cu}$ jet perforation diameter in the target plate was $19.6 \mathrm{~mm}$, while the PTFE jet was $17.4 \mathrm{~mm}$. The penetration aperture was increased by $12.6 \%$; however, its penetration capability was increased by $37.1 \%$, thus demonstrating quite significant improvement.

4. Experimental Studies on Kill Elements with Different Materials Penetrating Target. The process of the jet kill element penetrating target is a complex reaction process, and the numerical simulation cannot fully describe the real situation. In order to obtain a more realistic penetration effect, the kill element formation and the penetration into steel 45 armor by using the liner from three different materials and their combinations were experimentally tested under blast loading conditions. Figure 4 shows the experimental results of the kill element formation and the penetration into steel 45 armor. The $\mathrm{Cu}$ jet penetration depth was $97.5 \mathrm{~mm}$, and the perforation diameter was $12.1 \mathrm{~mm}$. The PTFE jet penetration depth was $19.0 \mathrm{~mm}$, and the perforation diameter was $18.3 \mathrm{~mm}$. PTFE-Cu jet penetration depth was $27.7 \mathrm{~mm}$, and the perforation diameter was $20.6 \mathrm{~mm}$. From Fig. 4 
$\mathrm{T}$ a b 1 e 3

A Comparison between the Numerical Simulation and Experimental Results

\begin{tabular}{|c|c|c|c|c|c|c|}
\hline \multirow{2}{*}{$\begin{array}{l}\text { Jet kill } \\
\text { element }\end{array}$} & \multirow{2}{*}{$\begin{array}{l}\text { Density } \\
\left(\mathrm{g} / \mathrm{cm}^{3}\right)\end{array}$} & \multirow{2}{*}{$\begin{array}{l}\text { Jet head } \\
\text { velocity } \\
(\mathrm{m} / \mathrm{s})\end{array}$} & \multicolumn{2}{|c|}{ Numerical simulation } & \multicolumn{2}{|c|}{ Experimental results } \\
\hline & & & $\begin{array}{l}\text { Penetration } \\
\text { depth }(\mathrm{mm})\end{array}$ & $\begin{array}{c}\text { Perforation } \\
\text { diameter }(\mathrm{mm})\end{array}$ & $\begin{array}{l}\text { Penetration } \\
\text { depth }(\mathrm{mm})\end{array}$ & $\begin{array}{c}\text { Perforation } \\
\text { diameter }(\mathrm{mm})\end{array}$ \\
\hline $\mathrm{Cu}$ & 8.93 & 4743 & 98.4 & 10.2 & 97.5 & 12.1 \\
\hline PTFE & 2.16 & 5575 & 22.1 & 17.4 & 19.0 & 18.3 \\
\hline PTFE-Cu & 3.05 & 5263 & 30.3 & 19.6 & 27.7 & 20.6 \\
\hline
\end{tabular}

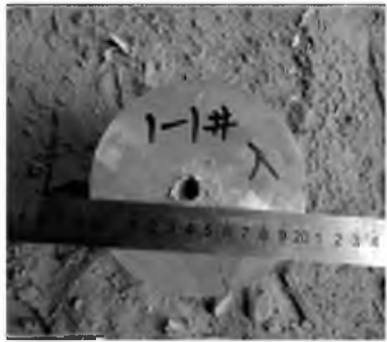

a

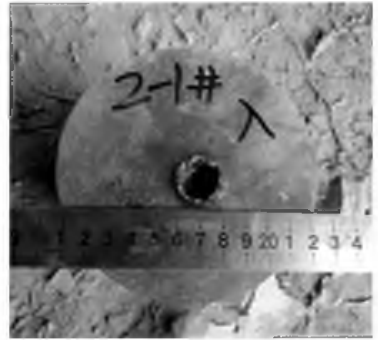

b

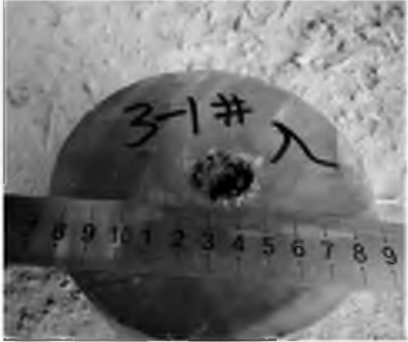

c

Fig. 4. The penetration results for kill elements from different materials: Cu (a), PTFE (b), and PTFE-Cu (c).

and Table 3, it can be seen that the experimental data are in good agreement with the numerical simulation results.

\section{Conclusions}

1. For the present study with the kill element formed by a combination of three different materials, numerical simulations and X-ray tests indicate that both PTFE-Cu and PTFE liners can form a good-shape jet kill element. The head velocity of the copper jet kill element was the smallest, whereas that of the PTFE one was the largest, with PTFE-Cu one exhibiting the in-between value.

2. Simulation results indicated that the head diameter of the PTFE-Cu jet kill element was $3 \mathrm{~mm}$, whereas that of the PTFE jet kill element was $2.7 \mathrm{~mm}$. Therefore, the former was improved by $11.1 \%$, as compared to the latter, and was twice as large as the head diameter of the copper jet kill element. Since the head diameter of the PTFE-Cu jet kill element was the largest, it demonstrated the highest level of reaming capability against the target, and could facilitate the opening of the channel for the subsequent stage in the tandem warhead.

3. For the experimental studies on the penetration performance of kill elements against steel armor when three different materials were applied, the penetration depth of the PTFE jet kill element was $19.0 \mathrm{~mm}$, whereas the penetration depth of the PTFE-Cu jet kill element was $27.7 \mathrm{~mm}$. Therefore, the penetration depth of the latter was improved by $45.8 \%$ as compared to the former. The perforation diameter of the PTFE jet kill element was $18.3 \mathrm{~mm}$, whereas that of the PTFE-Cu jet kill element was $20.6 \mathrm{~mm}$. Therefore, the perforation diameter of the latter was improved by $12.6 \%$ as compared to the former, indicating a high damage performance of the PTFE- $\mathrm{Cu}$ jet kill element.

Acknowledgment. The authors would like to acknowledge the financial support from Project supported by the National Natural Science Foundation of China under Grant No. 11572291. 
1. J. P. Yin and Z. J. Wang, Ammunition Theory, Beijing Institute of Technology Press (2014), pp.110-156.

2. C. Hong, New Advanced Technology of Ordnance Industry, Ordnance Industries Press (1994), pp. 319-320.

3. M. Held, "Liners for shaped charges," J. Battlefield Technol., 4, No. 3, 1-6 (2001).

4. A. Doig, "Some metallurgical aspects of shaped charge liners," J. Battlefield Technol., 1, No. 1, 1-3 (1998).

5. Z. W. Hu, Z. K. Li, T. J. Zhang, and X. M. Zhang, "Advanced progress in materials for shaped charge and explosively formed penetrator liners," Rare Metal Mat. Eng., 33, No. 10, 1009-1012 (2004).

6. Z. J. Guo, S. C. Zhang, and Y. Lin, "The development of material in shaped charges warhead liner," China Molybdenum Industry, 29, No. 4, 40-42 (2005).

7. C. Wu, S. S. Liao, T. Sun, et al., "Research on shaped charge warhead employing large diameter penetrating hole with tungsten alloy liner," Trans. Beijing Inst. Technol., 29, No. 9, 760-766 (2009).

8. B. H. Chang, J. P. Yin, Z. Q. Cui, and T. X. Liu, "Numerical simulation of modified low-density jet penetrating shell charge," Int. J. Simul. Model., 14, No. 3, 426-437 (2015).

9. M. Held, Initiation Criteria of High Explosives, Attacked with Projectiles of Different Densities (27th Int. Annual Conf. of ICT, June 25-28, 1996, Karlsruhe, Germany), TDW (1996), pp. 15-22.

10. X. Q. Ma, F. J. Zeng, M. L. Chen, et al., "A new approach anti-reactive armor," Acta Armamentaria, No. 4, 75-79 (1994).

11. F. D. Dong, Z. J. Wang, J. P. Yin, and S. P. Gao, "Numerical simulation on initiating value of low density jet impacting explosive with shell," Ordnance Mater. Sci. Eng., 36, No. 4, 53-55 (2013).

12. C. L. Zhao, Z. J. Wang, B. Cui, et al., "Numerical simulation of glass jet attacking shelled explosive," Ordnance Mater. Sci. Eng., 38, No. 3, 93-96 (2015).

13. M. Held, "Discussion of the experiment findings from the initiation of covered but unconfined high explosive charge with shaped jets," Propell. Explos. Pyrot., No. 12, 167-174 (1987).

14. B. H. Chang, J. P. Yin, Z. Q. Cui, and T. X. Liu, "Improved dynamic mechanical properties of modified PTFE jet penetrating charge with shell," Strength Mater., 48, No. 1, 82-89 (2016).

15. B. Li, Y. Fang, and P. F. Feng, "Process of damage research on shaped charge warhead of underwater weapon," J. Ordnance Equip. Eng., No. 2, 1-6 (2016). 